\title{
WF10 Stimulates NK Cell Cytotoxicity by Increasing LFA-1-Mediated Adhesion to Tumor Cells
}

\author{
Louisa Kühne, ${ }^{1,2}$ Mathias Konstandin, ${ }^{1,3}$ Yvonne Samstag, ${ }^{1}$ \\ Stefan Meuer, ${ }^{1}$ Thomas Giese, ${ }^{1}$ and Carsten Watzl ${ }^{1}$ \\ ${ }^{1}$ Institute for Immunology, University Heidelberg, Im Neuenheimer Feld 305, 69120 Heidelberg, Germany \\ ${ }^{2}$ Department of Nephrology, Klinikum Rechts der Isar, Technical University Munich, 81675 Munich, Germany \\ ${ }^{3}$ Department of Cardiology, University Heidelberg, 69120 Heidelberg, Germany
}

Correspondence should be addressed to Carsten Watzl, watzl@uni-hd.de

Received 24 January 2011; Accepted 25 February 2011

Academic Editor: Roberto Biassoni

Copyright (c) 2011 Louisa Kühne et al. This is an open access article distributed under the Creative Commons Attribution License, which permits unrestricted use, distribution, and reproduction in any medium, provided the original work is properly cited.

\begin{abstract}
The redox-active chlorite-based drug WF10 (Immunokine) was shown to have modulatory effects on both the innate and adaptive immune system in vitro and in vivo. Animal studies suggest that WF10 enhances immunity against tumors. One possible explanation for such an effect is that WF10 stimulates natural killer cell cytotoxicity against malignant cells. Here, we show that WF10 regulates human NK cell cytotoxicity in a time-dependent manner, following an S-shaped kinetic with an initial stimulation of activity followed by a decrease in activity relative to the untreated controls. WF10 does not activate NK cells on its own but costimulates NK cell activation mediated by different activating receptors. This is mediated by enhancing NK cell adhesion to target cells through promoting the activation of the integrin LFA-1. These data demonstrate a direct effect of WF10 on the cytotoxicity of human NK cells.
\end{abstract}

\section{Introduction}

Natural killer (NK) cells are important effector cells for innate immune reactions against viral infections and malignant cells [1]. NK cell effector functions include cellular cytotoxicity and the secretion of cytokines and chemokines. They also fulfill a regulatory role by engaging in cross-talk with diverse cellular components of the immune system [2]. NK cell cytotoxicity is regulated through the recognition of target cells by integrating positive and negative signals from activating and inhibitory receptor-ligand interactions [3]. Many inhibitory NK cell receptors are specific for selfMHC class I and are important to ensure the self-tolerance of NK cells. Loss of MHC class I upon viral infection or malignant transformation can, therefore, result in the socalled "missing-self" reactivity of NK cells. NK cell activation is mediated by a variety of different surface receptors that can recognize specific ligands on transformed or infected cells. Interestingly, freshly isolated resting human NK cells can only be activated by triggering two or more activating receptors in combination [4]. Therefore, all stimulatory receptors for natural cytotoxicity are considered to be coactivating. The only exception seems to be CD16, which is sufficient by itself to induce activation of resting NK cells.

NK cell adhesion to target cells is essential for enabling the interaction between NK cell receptors and their ligands within the immunological synapse and for the directed release of granules towards the attached target cell during NK cell cytotoxicity [5]. NK cell adhesion is mediated by integrins such as LFA-1. The function of LFA-1 is regulated by "inside-out signaling", where signals from other surface receptors influences the affinity of LFA-1 by inducing conformational changes of the molecule, and also affects the avidity of LFA-1 by driving mobility within the cell membrane [6]. This results in clustering of LFA-1 at the site of the NK cell synapse. Adhesion is, therefore, a highly regulated and dynamic process necessary for the function of NK cells. 
WF10, or Immunokine, is based on the chlorite ion matrix tetrachlorodecaoxygen and is currently being evaluated in various clinical indications including the adjuvant therapy of inoperable pancreatic cancer and various inflammatory conditions [7-9]. WF10 is known to have various immunological effects by stimulating innate immune functions, while inhibiting adaptive immune functions [10]. In contrast to control animals, sublethally irradiated rats treated with WF10 developed significantly less hematological or solid tumors than controls $[11,12]$. This antitumor effect of WF10 could be explained by stimulating the innate natural cytotoxicity against tumors. We, therefore, investigate the effect of WF10 on NK cell cytotoxicity.

\section{Materials and Methods}

2.1. Materials and Cells. WF10/Immunokine (containing ca. $63 \mu \mathrm{M}$ chlorite) was provided by Dimethaid $\mathrm{GmbH}$, Wanzleben, Germany. The following antibodies were used: MOPC21 (IgG1 isotype control, Sigma), antiNKp30 (p3015 , generated in our lab), antiNKp46 and antiCD16 (Beckmann Coulter), anti2B4 (Immunotech), antiNKG2D and antiDNAM-1 (R\&D Systems). Human ICAM-1-Fc was from R\&D Systems, PE-conjugated goat-antihuman $\mathrm{Fc} \gamma$ fragment-specific IgG $\mathrm{F}\left(\mathrm{ab}^{\prime}\right) 2$ fragment from Jackson Immuno Research. K562, LCL721.221 and P815 cells were maintained as described $[13,14]$. Human NK cells were isolated from PBMC by negative selection (Dynal, Invitrogen) and cultured as described [15].

2.2. Cytotoxicity Assay. For ${ }^{51} \mathrm{Cr}$ release assays, target cells were labeled with $100 \mu \mathrm{Ci}$ of ${ }^{51} \mathrm{Cr}$ for $1 \mathrm{~h}$ at $37^{\circ} \mathrm{C}$. The labeled cells were washed, mixed with NK cells at different effectorto-target $(\mathrm{E}: \mathrm{T})$ ratios on a 96-well V-bottom plate and incubated for $4 \mathrm{~h}$ at $37^{\circ} \mathrm{C}$. For redirected lysis assay NK cells were preincubated with antibodies $(0.5 \mu \mathrm{g} / \mathrm{ml}$ final concentration) for $15 \mathrm{~min}$ at $37^{\circ} \mathrm{C}$ before adding the target cells. The supernatant was harvested and ${ }^{51} \mathrm{Cr}$-release was measured in a $\gamma$-counter. Percent specific release was calculated as ( (experimental release-spontaneous release)/(maximum releasespontaneous release) $) \times 100$. The ratio between maximum and spontaneous release was at least three in all experiments.

2.3. Real-Time PCR Analysis. NK cells were incubated with or without WF10 for 3 or $18 \mathrm{~h}$. At the end of the incubation cells were lysed in $300 \mu \mathrm{l} \mathrm{MagNA}$ pure lysis buffer containing $1 \%$ DTT and mRNA was isolated using the MagnaPure-LC device. Isolated mRNA was transcribed into cDNA using AMV reverse transcriptase (First Strand cDNA synthesis kit (Roche)). Indicated primer sets (Search-LC, Heidelberg) were used with LightCycler-FastStart DNS Sybr Green I Kit (Roche) to amplify the cDNA using the LightCycler according to the manufacture's protocol. The number of transcripts of specific genes in each sample was normalised using the number of transcripts of the house-keeping genes $\beta$-actin and cyclophilin b. The transcript number was calculated from a virtual standard curve, obtained by plotting a known input concentration of a plasmid to the PCR cycle number (CP) at which the detected fluorescence intensity reaches a
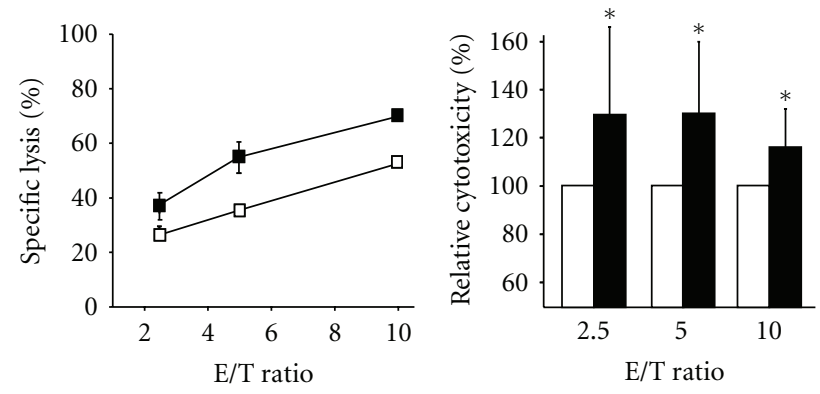

$$
\begin{aligned}
& \rightarrow-\text { Control } \\
& \rightarrow \text { WF10 }
\end{aligned}
$$

(a)

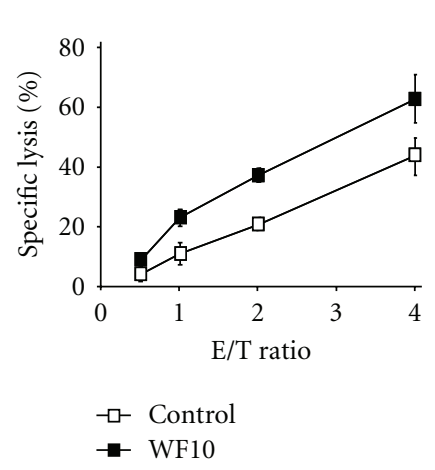

(c)

$$
\begin{aligned}
& \text { a Control } \\
& \text { - WF10 }
\end{aligned}
$$

(b)

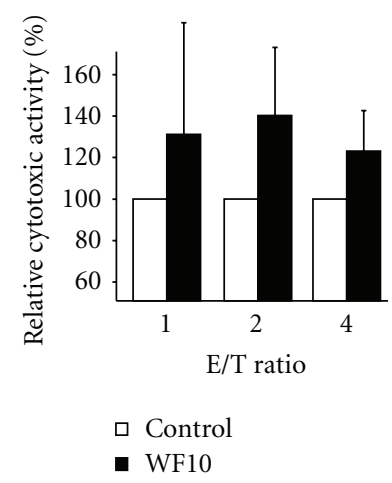

(d)
FIGURE 1: Effect of WF10 on the cytotoxic activity of NK cells. (a, b) Freshly isolated resting human NK cells or (c, d) IL-2 stimulated NK cells were used in a standard $4 \mathrm{~h}{ }^{51} \mathrm{Cr}$-release assays against LCL721.221 target cells with or without the addition of $200 \mu \mathrm{M}$ $\mathrm{WF} 10$ at the indicated effector to target (E/T) ratios. Panels (a) and (c) show the results of representative experiments. In panels (b) and (d) the activity of the WF10-treated cells is shown relative to the cytotoxic activity of control cells (set as 100\%) as an average value from (b) 24 or (d) 3 independent experiments. The ratio of each outcome and its control was tested against a value of 1 (equality) by two-sided single-sample $t$-tests $\left({ }^{*} P<.05\right)$.

fixed value. For better visualization, a $\log 2$ transformation, of the ratio between WF10-treated and control samples was calculated, as is common for gene expression studies [10].

\subsection{Conjugate Formation Assay and Ligand Complex-Based} Adhesion Assay (LC-AA). NK cell-target cell conjugate formation was measured by flow cytometry as described previously [13]. Briefly, freshly isolated NK cells were labeled with the dye PKH67 and LCL721.221 target cells with PKH26 (Sigma). Target and NK cells were combined and incubated with or without WF10 (final concentration of $200 \mu \mathrm{M}$ chlorite) at $37^{\circ} \mathrm{C}$. Reactions were stopped by vortexing, cells were fixed with ice-cold 4\% PFA and number of conjugates were determined by FACS analysis. The ligand-complexbased adhesion assay (LC-AA) assesses the activation of the adhesion molecule LFA-1 by FACS analysis of cell bound fluorescently-labeled ICAM-1-complexes and was performed as published $[16,17]$. For statistical analysis SPSS Statistics 17.0 was used. 


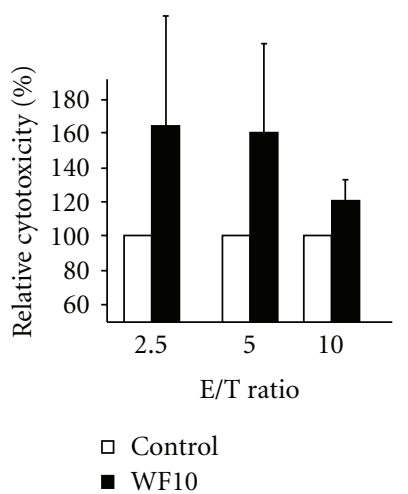

(a)

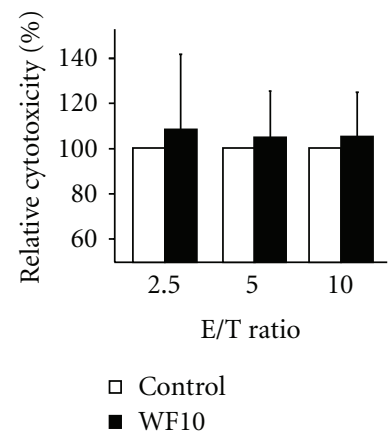

(b)

Figure 2: WF10 specifically affects NK cells. (a) Freshly isolated resting human NK cells were preincubated for $5 \mathrm{~h}$ with or without $200 \mu \mathrm{M}$ WF10 in culture medium, washed and then used in a standard $4 \mathrm{~h}{ }^{51} \mathrm{Cr}$-release assays against LCL721.221 target cells at the indicated effector to target (E/T) ratios. (b) LCL721.221 target cells were preincubated with or without $200 \mu \mathrm{M}$ WF10 in culture medium, washed and then used as targets in a standard $4 \mathrm{~h}{ }^{51} \mathrm{Cr}$ release assays using freshly isolated human NK cells as effectors. The activity of the WF10-treated cells is shown relative to the cytotoxic activity of control cells (set as 100\%) as an average value from (a) 4 or (b) 3 independent experiments.

\section{Results and Discussion}

3.1. WF10 Can Increase the Cytotoxic Activity of Human NK Cells. To test whether WF10 is able to boost the cytotoxic activity of NK cells, we used freshly isolated human NK cells in a standard $4 \mathrm{~h}{ }^{51} \mathrm{Cr}$-release assay against the MHC class I-negative B cell line LCL721.221. At a therapeutic concentration of $200 \mu \mathrm{M}$ active chlorite content WF10 significantly enhanced the cytotoxic activity of NK cells (Figures $1(\mathrm{a})$ and 1(b)). This effect was also seen when PBMC (data not shown) and IL-2-activated human NK cells were used (Figures 1(c) and $1(\mathrm{~d})$ ). The enhancement of the NK cell cytotoxicity by WF10 was dose-dependent and could also be observed when NK cells were pretreated with WF10 before the assay (Figure 2(a)). However, WF10 did not directly affect the viability of target cells and pretreatment of target cells with WF10 did not alter their susceptibility to NK-mediated lysis (Figure 2(b)). These data demonstrate that WF10 specifically enhances the cytotoxic activity of NK cells. This effect was not restricted to 721.221 target cells, but also the lysis of the leukemic cell line K562 and the pancreatic cancer cell line Miapaca were enhanced by WF10 (data not shown).

3.2. Time-Dependent Effect of WF10. The increase in NK cell cytotoxicity by WF10 was time-dependent, following an Sshaped kinetic over 24 hours (Figure 3). After an initial boost of activity, WF10 inhibited NK cell cytotoxicity after $18 \mathrm{~h}$ of pretreatment (Figures 3(b) and 3(c)). Preincubation of NK cells for 24 hours did no longer result in differences of cytotoxicity between WF10 or control treated cells. The inhibition of NK cell cytotoxicity was not due to a cytotoxic effect of WF10 as we only detected a minor increase in NK cell

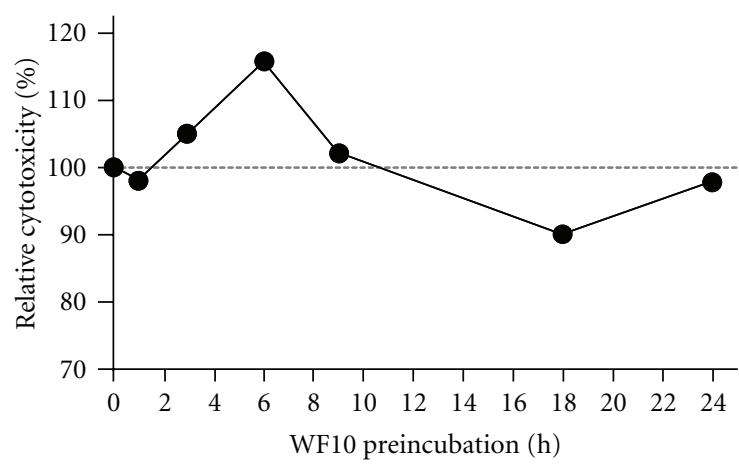

(a)

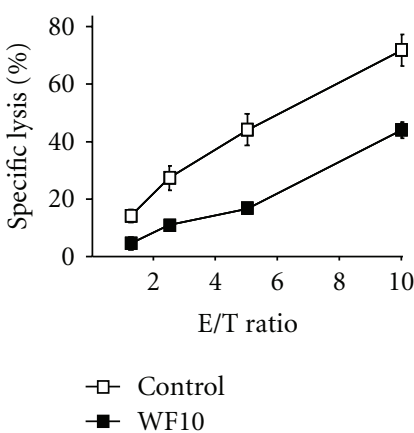

(b)

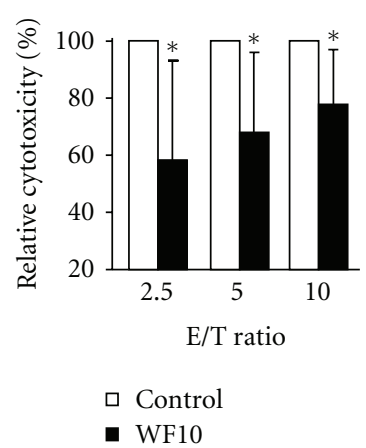

(c)
Figure 3: Time-dependent modulation of NK cell responses by WF10. (a) Freshly isolated resting human NK cells or were preincubated for the indicated times with $200 \mu \mathrm{M}$ WF10 and then used in a standard $4 \mathrm{~h}{ }^{51} \mathrm{Cr}$-release assays against LCL721.221 target cells. Cytotoxic activity was normalized to the untreated control (set as 100\%). Shown are the average values of two independent experiments using NK cells from different donors. (b, c) Freshly isolated resting human NK cells were incubated with or without $200 \mu \mathrm{M}$ WF10 in culture medium for $18 \mathrm{~h}$. Cells were then resuspended in fresh medium and tested as described above. Panel (b) shows the results of a representative experiment. In panel (c) the activity of the WF10-treated cells is shown relative to the cytotoxic activity of control cells (set as 100\%) as an average value from 8 independent experiments. The ratio of each outcome and its control was tested against a value of 1 (equality) by two-sided single-sample $t$-tests $\left({ }^{*} P<.05\right)$.

apoptosis after $18 \mathrm{~h}$ of WF10-treatment. However, consistent with previous findings that WF10 affects gene transcription by modulating certain transcription factors in PBMC [10], we detected a reduced expression of cytotoxicity-related genes such as NKG2D, Perforin and DAP12 by quantitative RT-PCR after $18 \mathrm{~h}$ of WF10-treatment (Figure 4), which might explain the reduction in cytotoxicity.

3.3. WF10 Enhances NK Cell Cytotoxicity Mediated by Different Activating Receptors. Next we wanted to test if the WF10-mediated enhancement of NK cell activity was mediated through a specific activating receptor. Therefore, we triggered NK cell cytotoxicity through NKp30, NKG2D or $2 \mathrm{~B} 4$ in a redirected lysis assay. In line with the concept of NK cell coactivation [4], triggering of any of these 


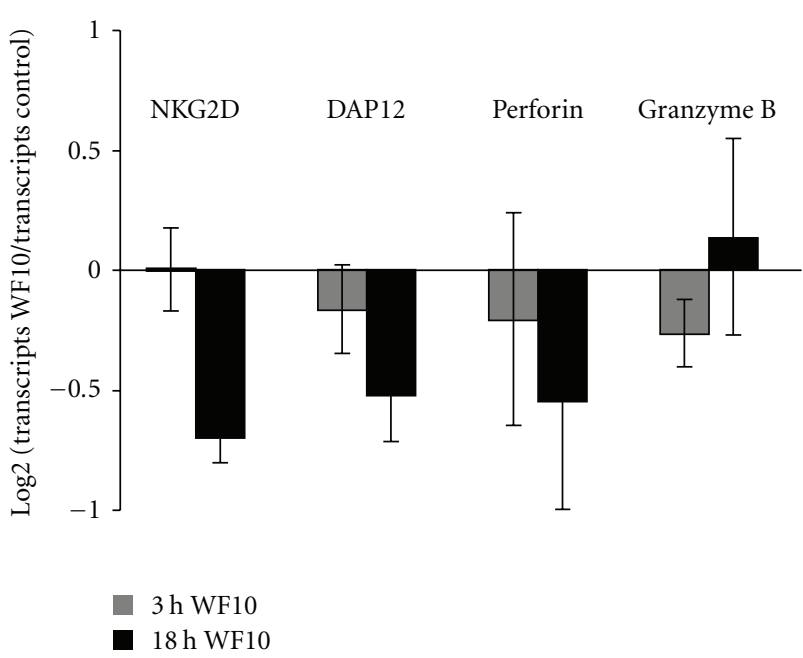

FIGURE 4: The effect of WF10 on the transcription of NK cell genes. Freshly isolated NK cells were incubated with WF10 (at a chlorite concentration of $200 \mu \mathrm{M}$ ) for 0,3 , or $18 \mathrm{~h}$ and mRNA was isolated. The indicated transcripts were quantified by quantitative RT-PCR. The number of transcripts of individual genes in each sample was normalised using the number of transcripts of the house-keeping genes $\beta$-actin and cyclophilin b. The number of transcripts in the WF10-treated sample was compared with the number of transcripts in the control sample using the formula $\log 2$ (transcripts WF10 sample/transcripts control sample). Average values and standard deviation from 5 independent experiments with different blood donors are shown.

receptors on freshly isolated NK cells did not stimulate cytotoxicity. Also the addition of WF10 did not change this. However, when we used IL-2-activated NK cells, WF10 could enhance NK cell cytotoxicity mediated by CD16, NKG2D, NKp30 and 2B4 (Figure 5). This suggests that WF10 acts independently of one specific receptor proximal signaling pathway. Interestingly, WF10 can only costimulate NK cell cytotoxicity but does not lead to NK cell activation on its own.

3.4. WF10 Enhances Target Cell Adhesion and Co-Stimulates the Activation of LFA-1. As WF10 acted independently of specific receptor signaling pathways, we tested its effect on NK cell adhesion to target cells. This adhesion is essential for NK cell cytotoxicity. It allows the interaction of NK cell receptors with their ligands on the target cells and is essential for the directed release of cytotoxic granules. During the first 90 minutes of conjugate formation between freshly isolated NK cells and 721.221 target cells WF10 showed only a minor effect. However, at later time points WF10treatment resulted in an increase in the amount of conjugates (Figure 6(a)). LFA-1 is an important adhesion molecule on NK cells. The affinity and avidity of LFA-1 for its ligand ICAM-1 is regulated by inside-out-signals that can derive from cytokines or the activation of cytotoxicity receptors [6]. We, therefore, tested the binding activity of LFA-1 using a ligand-complex-based adhesion assay (LC-AA) [16]. In this assay fluorescently-labeled ICAM-1 complexes are used for the staining of cells in a FACS-based analysis. These

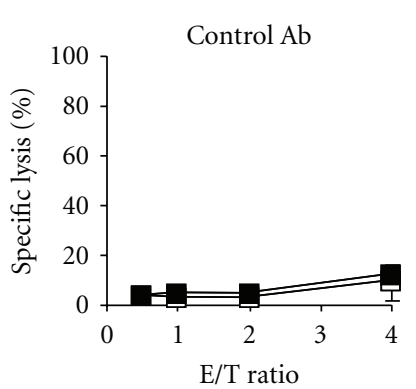

(a)

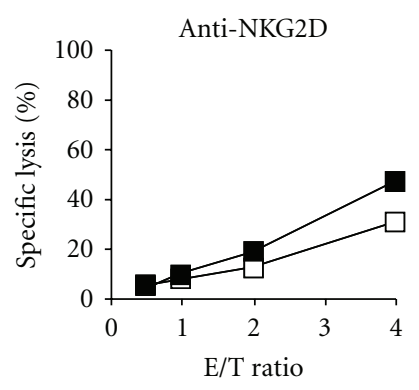

(c)

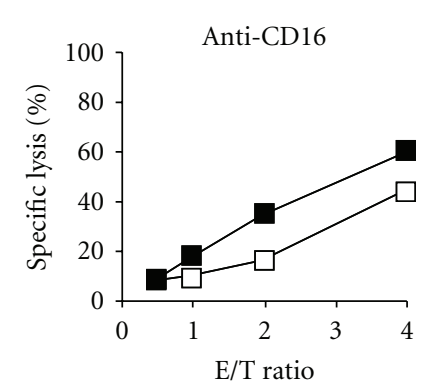

(b)

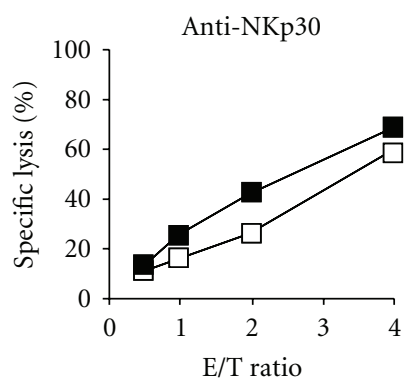

(d)

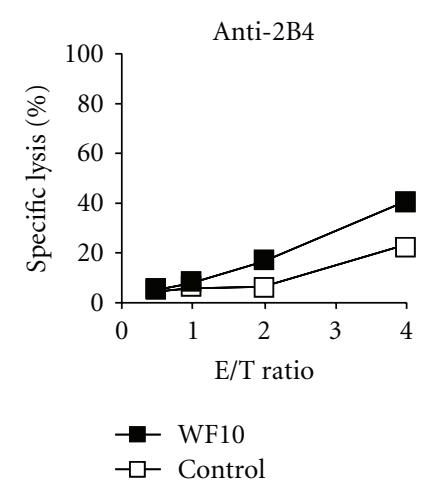

(e)

FIGURE 5: Enhancement of receptor-mediated cytotoxicity of IL2-activated NK cells by WF10. IL-2-activated NK cells were tested in a redirected-lysis assay against murine P815 cells. NK cells were stimulated using a (a) control antibody, or by antibodies directed against the activating receptors (b) CD16, (c) NKG2D, (d) NKp30, or (e) 2B4. Where indicated, WF10 was added to the assay medium at a final chlorite concentration of $200 \mu \mathrm{M}$. Average values of triplicates are shown with the standard deviation. One representative of three independent experiments with different donors is shown.

complexes will only bind to LFA-1 in its high affinity or high avidity conformation. Freshly isolated NK cells did not show binding of ICAM-1 (Figures 6(b) and 6(c)), indicating that LFA-1 is not activated in resting NK cells. The addition of WF10 did not change this, which confirms that WF10 cannot stimulate NK cell activation by its own, but can only costimulate other activation signals. To induce inside-out signaling we stimulated the NK cells with IL-15, which resulted in LFA-1 activation (Figure 6(b)). WF10 significantly increased the LFA-1 activation after IL-15 incubation (Figures 6(b) and 6(c)). These data suggest that WF10 can enhance NK cell 


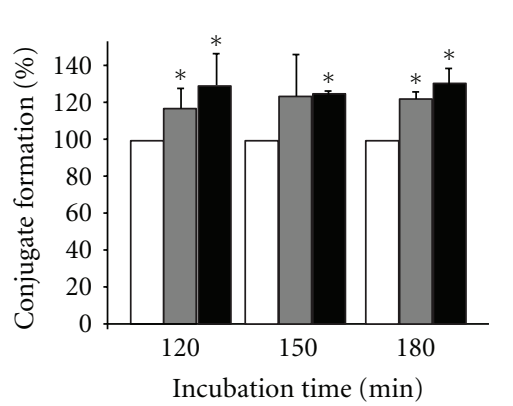

$\square$ Control -WF10 $(600 \mu \mathrm{M})$
$\square \operatorname{WF10}(200 \mu \mathrm{M})$

(a)
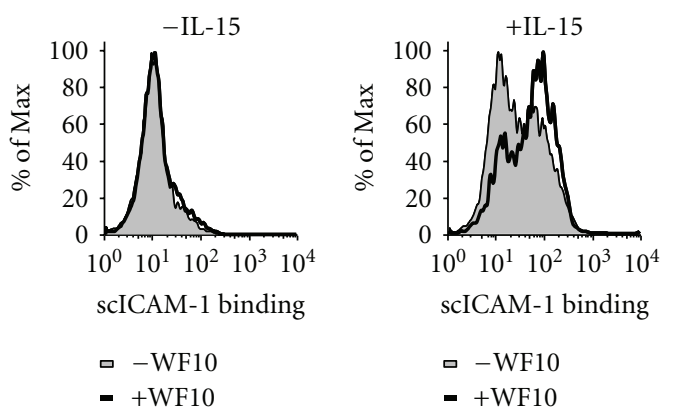

(b)

FIGURE 6: WF10 enhances NK cell adhesion. (a) NK cells and 721.221 cells were labeled with a different fluorescent dye, washed, and then incubated together with or without WF10 (200 or $600 \mu \mathrm{M})$. At the indicated time points the mixed suspension was fixed with ice-cold $4 \%$ PFA and analyzed by FACS. The amount of NK: 721.221 conjugates formed by the cells without WF10 was used as a reference $(100 \%)$. Average values and the standard deviation from 3 independent experiments with different blood donors are shown. (b, c) Freshly isolated NK cells were incubated with PEconjugated ICAM-1 complex (scICAM-1) with or without WF10 for $3 \mathrm{~h}$. Samples were either left unstimulated (b, left panel), or treated with IL-15 (100 U/ml) (b, right panel). The amount of bound ICAM-1 complexes was measured by FACS. A representative experiment is shown in (b). The ICAM-1 binding to control cells (MFI $44.2 \pm 20.4)$ and IL-15 stimulated cells (MFI $126 \pm 60.3$ ) was used as a reference (100\%). (c) Average values and standard deviations from 5 independent experiments with different blood donors are shown. The ratio of each outcome and its control was tested against a value of 1 (equality) by two-sided single-sample $t$ tests $\left({ }^{*} P<.05\right)$.

adhesion to target cells by costimulating inside-out signaling resulting in higher LFA-1 activity. This would explain the higher cytotoxic activity of NK cells after WF10-treatment and suggests that rather than hyperactivating a few NK cells to kill more frequently, WF10 may increase the number of NK cells involved in cytotoxicity at a given time, so that cells that were previously insufficiently activated are now recruited to the killing process by WF10. This would be in line with our finding that WF10 can costimulate NK cells, but not activate them on its own.

\section{Concluding Remarks}

WF10 was shown to inhibit the development and promotion of leukemia in animal models $[11,12]$. The role of oxidative species in cancer is complex and has been extensively studied in the last decades. ROS appear to have a dual role in tumor development and growth. Oxidative species contribute to DNA damage, a major step in carcinogenesis and progression, but they can also act in pro-apoptotic signaling pathways and, therefore, have antitumor effects [18]. Due to this dual role, some anticancer strategies are based on pro-oxidant mechanisms and some on antioxidant mechanisms [19]. In addition to these direct effects, we have shown in this study that WF10 can stimulate NK cell cytotoxicity through promoting LFA-1-mediated adhesion to tumor cells. As NK cells are known to be important effector cells against hematological malignancies [20], it may be promising to combine WF10 with other strategies of NK cellbased immunotherapy against cancer. However, the effects of WF10 on immune function appears to be modulatory, rather than either stimulatory or inhibitory. It is, therefore, expected that a treatment with WF10 could only temporary enhance NK cell functions, followed by a phase of reduced NK cell activity. It is unclear if this transient inhibition of NK cell cytotoxicity, possibly mediated by the downregulation of cytotoxicity-related genes such as NKG2D, DAP12, or perforin, would lead to a gap in immune defense mechanisms. However, this transient inhibition of NK cell cytotoxicity may be overcome by combining the WF10-based therapy with stimulatory substances, such as Interleukin15 , for which a synergistic effect in combination with WF10 has been shown in our study. Additionally, our data would suggest that it may be beneficial to administer WF10 in individual boosts rather than maintaining a constant drug level in order to exploit its influence on NK cell activity.

\section{Author Contribution}

L. Kühne performed research; M. Konstandin and Y. Samstag helped with LC-AA binding assay; L. Kühne, S. Meuer, T. Giese and C. Watzl designed research and analyzed the data; C. Watzl and L. Kühne wrote the manuscript.

\section{Abbreviations \\ LC-AA: Ligand-complex-based adhesion assay, \\ LFA-1: Lymphocyte function-associated antigen-1, \\ NK: Natural killer, \\ PBMC: peripheral blood mononuclear cells.}

\section{Acknowledgments}

The would like to thank Birgitta Messmer for her support in isolating and culturing human NK cells. This work was supported by the BioFuture Program of the BMBF (to C. Watzl). 


\section{References}

[1] E. Vivier, E. Tomasello, M. Baratin, T. Walzer, and S. Ugolini, "Functions of natural killer cells," Nature Immunology, vol. 9, no. 5, pp. 503-510, 2008.

[2] A. Moretta, "Natural killer cells and dendritic cells: rendezvous in abused tissues," Nature Reviews Immunology, vol. 2, no. 12, pp. 957-964, 2002.

[3] L. L. Lanier, "Up on the tightrope: natural killer cell activation and inhibition," Nature Immunology, vol. 9, no. 5, pp. 495-502, 2008.

[4] Y. T. Bryceson, M. E. March, H. G. Ljunggren, and E. O. Long, "Synergy among receptors on resting NK cells for the activation of natural cytotoxicity and cytokine secretion," Blood, vol. 107, no. 1, pp. 159-166, 2006.

[5] J. C. Stinchcombe and G. M. Griffiths, "Secretory mechanisms in cell-mediated cytotoxicity," Annual Review of Cell and Developmental Biology, vol. 23, pp. 495-517, 2007.

[6] B. H. Luo, C. V. Carman, and T. A. Springer, "Structural basis of integrin regulation and signaling," Annual Review of Immunology, vol. 25, pp. 619-647, 2007.

[7] I. A. Malik, I. Moid, S. Haq, and M. Sabih, "A double-blind, placebo-controlled, randomized trial to evaluate the role of tetrachlorodecaoxide in the management of chemotherapyinduced oral mucositis," Journal of Pain and Symptom Management, vol. 14, no. 2, pp. 82-87, 1997.

[8] V. Veerasarn, W. Boonnuch, and C. Kakanaporn, "A phase II study to evaluate WF10 in patients with late hemorrhagic radiation cystitis and proctitis," Gynecologic Oncology, vol. 100, no. 1, pp. 179-184, 2006.

[9] S. Penpattanagul, "Reduced incidence and severity of acute radiation mucositis by WF10 (IMMUNOKINE) as adjunct to standard of cure in the management of head \& neck cancer patients," Journal of the Medical Association of Thailand, vol. 90, no. 8, pp. 1590-1600, 2007.

[10] T. Giese, M. S. McGrath, S. Stumm, H. Schempp, E. Elstner, and S. C. Meuer, "Differential effects on innate versus adaptive immune responses by WF10," Cellular Immunology, vol. 229, no. 2, pp. 149-158, 2004.

[11] S. R. Kempf, K. Blaszkiewitz, R. E. Port, and S. Ivankovic, "Influence of tetrachlorodecaoxide (Ryoxon) on the development of leukemia after total-body gamma-irradiation," Oncology, vol. 51, no. 6, pp. 510-514, 1994.

[12] S. R. Kempf, R. E. Port, and S. Ivankovic, "Anticarcinogenic effect of tetrachlorodecaoxide after total-body gamma irradiation in rats," Radiation Research, vol. 139, no. 2, pp. 226-231, 1994.

[13] P. C. Raemer, K. Kohl, and C. Watzl, "Statins inhibit NKcell cytotoxicity by interfering with LFA-1-mediated conjugate formation," European Journal of Immunology, vol. 39, no. 6, pp. 1456-1465, 2009.

[14] R. Bhat and C. Watzl, "Serial killing of tumor cells by human natural killer cells-enhancement by therapeutic antibodies," PLoS ONE, vol. 2, no. 3, article e326, 2007.

[15] P. Eissmann and C. Watzl, "Molecular analysis of NTB-A signaling: a role for EAT-2 in NTB-A-mediated activation of human NK cells," Journal of Immunology, vol. 177, no. 5, pp. 3170-3177, 2006.

[16] M. H. Konstandin, U. Sester, M. Klemke, T. Weschenfelder, G. H. Wabnitz, and Y. Samstag, "A novel flow-cytometrybased assay for quantification of affinity and avidity changes of integrins," Journal of Immunological Methods, vol. 310, no. 1-2, pp. 67-77, 2006.
[17] S. C. Hoffmann, A. Cohnen, T. Ludwig, and C. Watzl, “2B4 engagement mediates rapid LFA-1 and actin-dependent NK cell adhesion to tumor cells as measured by single cell force spectroscopy," Journal of Immunology, vol. 186, no. 5, pp. 2757-2764, 2011.

[18] M. Valko, C. J. Rhodes, J. Moncol, M. Izakovic, and M. Mazur, "Free radicals, metals and antioxidants in oxidative stressinduced cancer," Chemico-Biological Interactions, vol. 160, no. 1, pp. 1-40, 2006.

[19] J. Wang and J. Yi, "Cancer cell killing via ROS: to increase or decrease, that is a question," Cancer Biology and Therapy, vol. 7, no. 12, pp. 1875-1884, 2008.

[20] M. Terme, E. Ullrich, N. F. Delahaye, N. Chaput, and L. Zitvogel, "Natural killer cell-directed therapies: moving from unexpected results to successful strategies," Nature Immunology, vol. 9, no. 5, pp. 486-494, 2008. 


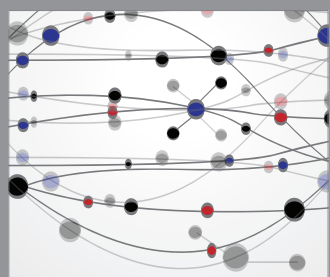

The Scientific World Journal
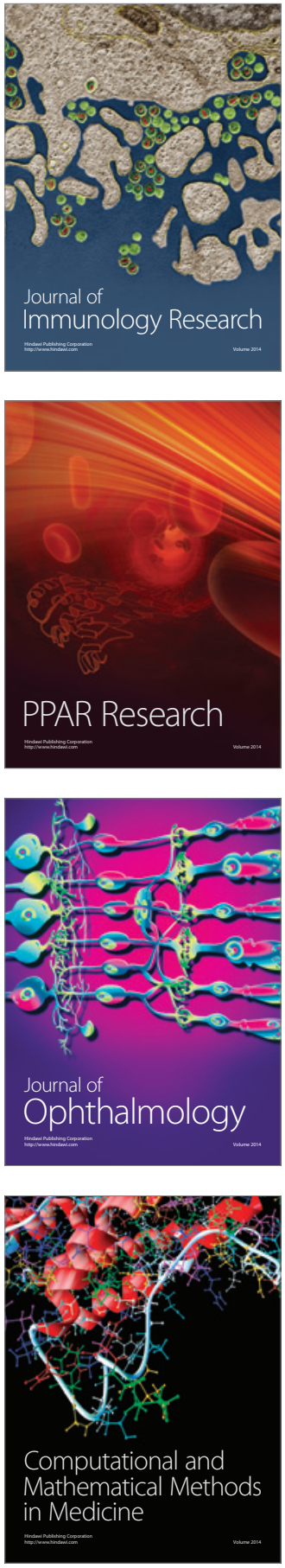

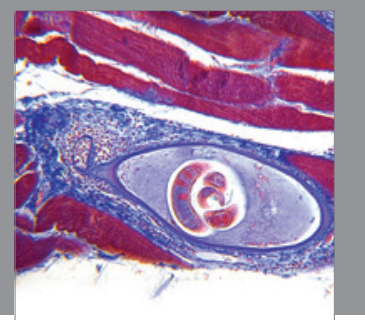

Gastroenterology

Research and Practice
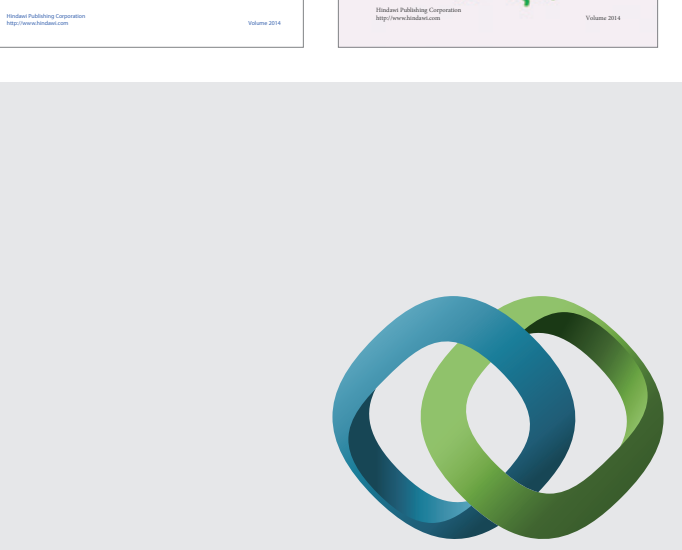

\section{Hindawi}

Submit your manuscripts at

http://www.hindawi.com
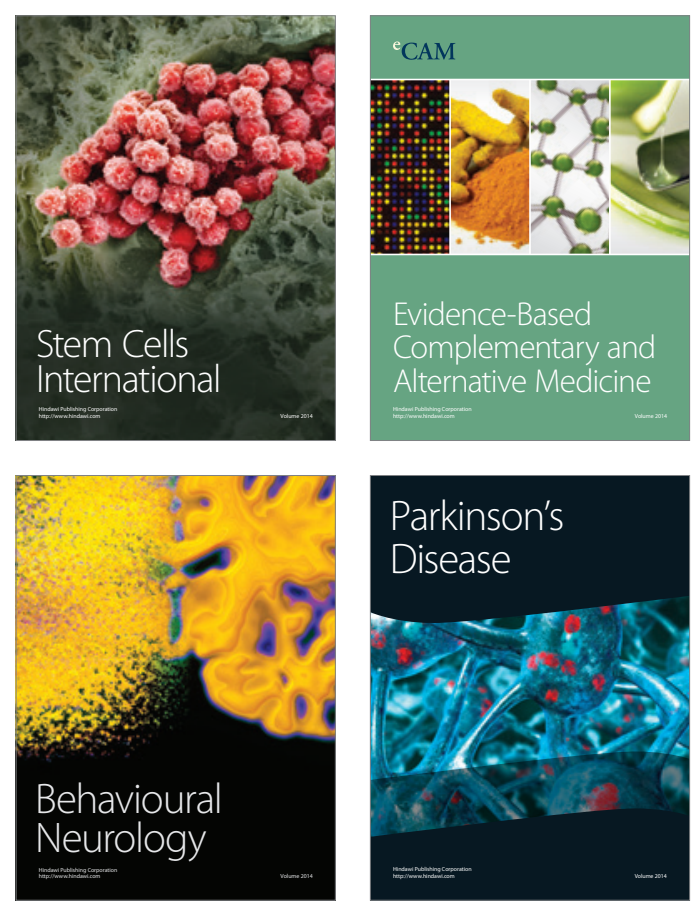

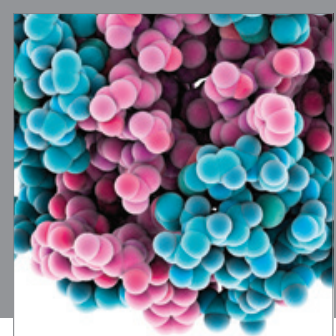

Journal of
Diabetes Research

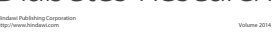

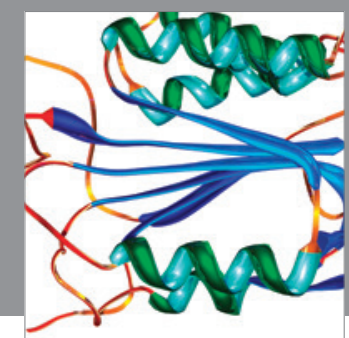

Disease Markers
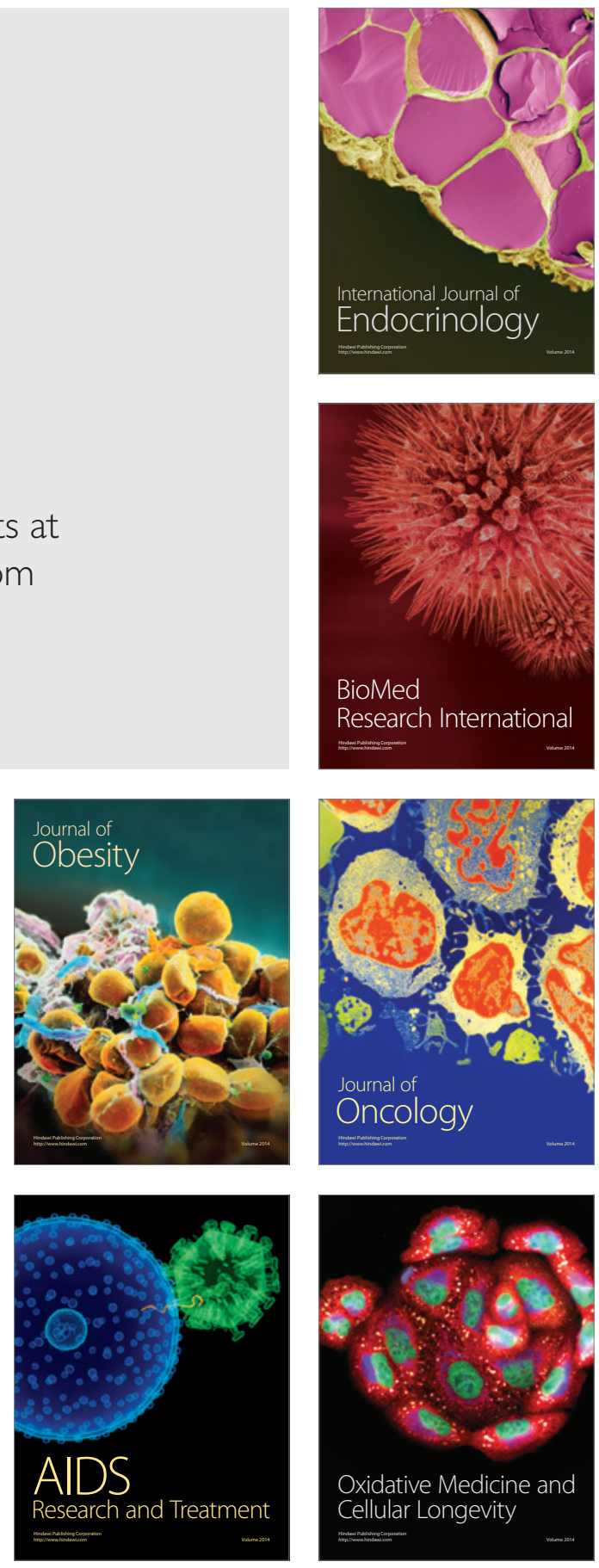